\title{
Rodent Malaria Erythrocyte Preference Assessment by an Ex Vivo Tropism Assay
}

\author{
Yew Wai Leong ${ }^{1,2,3}$, Erica Qian Hui Lee ${ }^{3}$, Laurent Rénia ${ }^{1,2,4^{*}+}$ and Benoit Malleret ${ }^{2,3 *}$ \\ ${ }^{1}$ Agency for Science, Technology and Research Infectious Diseases Laboratories (A*STAR ID Labs), Immunos, Biopolis, \\ Singapore, Singapore, ${ }^{2}$ Singapore Immunology Network, Agency for Science, Technology and Research (A*STAR), \\ Immunos, Biopolis, Singapore, Singapore, ${ }^{3}$ Department of Microbiology and Immunology, Immunology Translational \\ Research Program, Yong Loo Lin School of Medicine, Immunology Program, Life Sciences Institute, National University of \\ Singapore (NUS), Singapore, Singapore, ${ }^{4}$ Lee Kong Chian School of Medicine, Nanyang Technological University, \\ Singapore, Singapore
}

OPEN ACCESS

Edited by:

Bruce Malcolm Russell,

University of Otago, New Zealand

Reviewed by:

Renato Augusto DaMatta,

State University of the North

Fluminense Darcy Ribeiro, Brazil Shannon Moonah,

University of Virginia, United States

${ }^{*}$ Correspondence: Laurent Rénia

renia_laurent@idlabs.a-star.edu.sg

Benoit Malleret

benoit_malleret@nus.edu.sg

${ }^{\dagger}$ These authors have contributed equally to this work and share last authorship

Specialty section: This article was submitted to Parasite and Host, a section of the journal

Frontiers in Cellular and Infection Microbiology

Received: 13 March 2021 Accepted: 22 June 2021 Published: 12 July 2021

Citation: Leong YW, Lee EQH, Rénia L and Malleret B (2021) Rodent Malaria Erythrocyte Preference Assessment by an Ex Vivo Tropism Assay. Front. Cell. Infect. Microbiol. 11:680136. doi: 10.3389/fcimb.2021.680136
Circulating red blood cells consist of young erythrocytes (early and late reticulocytes) and mature erythrocytes (normocytes). The human malaria parasites, Plasmodium falciparum and $P$. vivax, have a preference to invade reticulocytes during blood-stage infection. Rodent malaria parasites that also prefer reticulocytes could be useful tools to study human malaria reticulocyte invasion. However, previous tropism studies of rodent malaria are inconsistent from one another, making it difficult to compare cell preference of different parasite species and strains. In vivo measurements of cell tropism are also subjected to many confounding factors. Here we developed an ex vivo tropism assay for rodent malaria with highly purified fractions of murine reticulocytes and normocytes. We measured invasion into the different erythrocyte populations using flow cytometry and evaluated the tropism index of the parasite strains. We found that $P$. berghei ANKA displayed the strongest reticulocyte preference, followed by $P$. yoelii $17 \times 1$ 1, whereas $P$. chabaudi AS and $P$. vinckei S67 showed mixed tropism. These preferences are intrinsic and were maintained at different reticulocyte and normocyte availabilities. Our study shed light on the true erythrocyte preference of the parasites and paves the way for future investigations on the receptor-ligand interactions mediating erythrocyte tropism.

Keywords: erythrocyte tropism, reticulocyte, normocyte, erythrocyte invasion, rodent malaria, flow cytometry

\section{INTRODUCTION}

Despite being enucleated, circulating erythrocytes are phenotypically diverse and range from young erythrocytes (which consist of early and late reticulocytes) to fully matured biconcave erythrocytes (normocytes). Early reticulocytes egress out of hematopoietic organs (primarily the bone marrow) into the peripheral circulation, where they only consist around 1-3\% of total human erythrocytes (Chin-Yee et al., 1991). Human reticulocytes undergo extensive cellular changes as they complete their maturation into normocytes (Malleret et al., 2013), such as the loss of ribosomal RNA and the downregulation of many surface proteins (Wilson et al., 2016; Chu et al., 2018; Gautier et al., 2018).

The causative agent of malaria, Plasmodium spp., parasitizes on hepatocytes and erythrocytes of the vertebrate host. During blood-stage infection, parasite entry into erythrocytes is mediated by 
interactions between various parasite invasion ligands and their cognate erythrocyte surface receptors (Cowman et al., 2017). P. vivax, the most widespread human malaria species, has a stringent erythrocyte tropism and can only invade the most immature human reticulocytes (Malleret et al., 2015). It is thought that the reason behind this strict tropism is because $P$. vivax can only invade via receptors that are only present on reticulocytes, and indeed, two such reticulocyte-specific receptors, CD71 and CD98, were identified recently (Gruszczyk et al., 2018; Malleret et al., 2021). P. falciparum, the deadliest human malaria species, also has a preference for reticulocytes, but can invade normocytes too (Pasvol et al., 1980; Naidu et al., 2019). However, no reticulocyte-specific receptor for $P$. falciparum invasion has been identified so far (Cowman et al., 2017).

Due to obvious challenges of studying human malaria parasites, mouse models of malaria have always been an important part of malaria research (Zuzarte-Luis et al., 2014; De Niz and Heussler, 2018). There is some degree of conservation in the invasion pathways across different human and rodent malaria species, in terms of utilization of homologous parasite ligands (Iyer et al., 2007; Gunalan et al., 2013) and erythrocyte receptors (Swardson-Olver et al., 2002). This makes reticulocyte-preferring rodent malaria viable models to study human malaria reticulocyte invasion.

Historically, the erythrocyte tropism of rodent malaria parasites was studied by microscopy on blood samples of infected animals. From these studies, it is generally accepted that $P$. berghei ANKA and non-lethal strains of $P$. yoelii have a preference for reticulocytes (Fahey and Spitalny, 1984; Deharo et al., 1996; Cromer et al., 2006; Otsuki et al., 2009), whereas $P$. vinckei has a tendency to invade normocytes (Viens et al., 1971; Vigário et al., 2001). Another rodent malaria parasite, $P$. chabaudi, was reported to have a mixed tropism and equally prefers both reticulocytes and normocytes (Jarra and Brown, 1989). These studies, however, differ from one another in their ways of measuring erythrocyte preference, making tropism comparisons between different parasite species difficult. Microscopic evaluations are also subjective, and this problem is compounded by the difficulty of differentiating late-stage parasites in reticulocytes from those in normocytes. More recent tropism studies used flow cytometry to distinguish erythrocyte types by their levels of CD71 expression, but these are constrained to only certain species, like P. berghei (Dertinger et al., 2000; Thakre et al., 2018).

More importantly, in vivo tropism measurements have limitations in determining the true erythrocyte preference. First and foremost, blood samples of infected animals are only a 'timepoint snapshot' of erythrocyte types and do not represent the erythrocyte stage during the point of merozoite invasion. This problem is exacerbated by the potential acceleration of reticulocyte maturation after invasion, as seen in $P$. vivax (Malleret et al., 2015). Additionally, studies with $P$. vivax and $P$. yoelii have shown that infected reticulocytes are more prone to removal by the host's cytotoxic CD8+ T-cells (Junqueira et al., 2018; Hojo-Souza et al., 2020), resulting in the underrepresentation of infected reticulocytes in circulation. Peripheral blood data are also made even more unreliable considering the extent of organ and deep vasculature sequestration of infected cells (Franke-Fayard et al., 2010; Claser et al., 2011), and the presence of extravascular parasite populations in hematopoietic organs (De Niz et al., 2018; Lee et al., 2018).

Here we comprehensively investigated the erythrocyte tropism of the four major rodent malaria species ( $P$. berghei, $P$. yoelii, $P$. chabaudi, and $P$. vinckei) in vivo and ex vivo. To study their tropism in vivo, we used flow cytometry to clearly define infected reticulocytes and normocytes based on the expressions of reticulocyte-specific markers, CD71 and CD98. Realizing the need for a way to measure tropism without the influence of the above-mentioned in vivo confounders, we also developed an ex vivo flow cytometry-based tropism assay. Late-stage parasites were exposed to highly purified fractions of mouse reticulocytes and normocytes, and new invasions into each erythrocyte type were compared. The assay allows for a more accurate evaluation of the parasites' intrinsic erythrocyte preference and crossspecies comparisons.

\section{RESULTS}

\section{In Vivo Tropism of Rodent Malaria}

To define mouse reticulocytes and normocytes in vivo, we utilized CD71 and CD98 as reticulocyte-specific markers. Circulating erythrocytes in naïve mice can be divided into three main populations: CD71+ CD98+, CD71+ CD98-, and CD71- CD98- erythrocytes. Based on their RNA content measured by thiazole orange staining, CD71+ CD98+ cells are the early reticulocytes, CD71+CD98-cells are late reticulocytes, and CD71- CD98- cells are mainly normocytes (Supplementary Figures S1A, B). Interestingly, in malaria-infected mice, there also exists a population of CD71- CD98+ erythrocytes (Supplementary Figure S1C), whose role and relevance in the infection is still under investigation. For simplicity and to have a clear distinction between reticulocytes and normocytes, here we only focus on the CD71+CD98+ reticulocytes and CD71- CD98normocytes to measure erythrocyte tropism. Using this definition of erythrocyte types, we compared the erythrocyte tropism of the four rodent malaria species. These parasites strains have different parasitemia profiles in C57BL/6 mice (Supplementary Figure S2), so we compared erythrocyte tropism at two timepoints before the peak of infection for each strain. For the lethal strains, $P$. berghei ANKA (PbA) and $P$. vinckei vinckei S67 (PvvS67), we measured tropism before the mice started to succumb to the infection. For the self-resolving strains, P. yoelii 17X1.1 (Py1.1) and P. chabaudi chabaudi AS (PccAS), we measured tropism before the peak of parasitemia.

Before the peak of $\mathrm{PbA}$ infection, there were more infected normocytes than infected reticulocytes in circulation (Figure 1A), which could imply that $\mathrm{PbA}$ has a normocyte preference. However, normocytes were far more abundant than reticulocytes in circulation during this period (Supplementary Figure S3). This means that $\mathrm{PbA}$ merozoites were more likely to 
$\mathrm{PbA}$

A

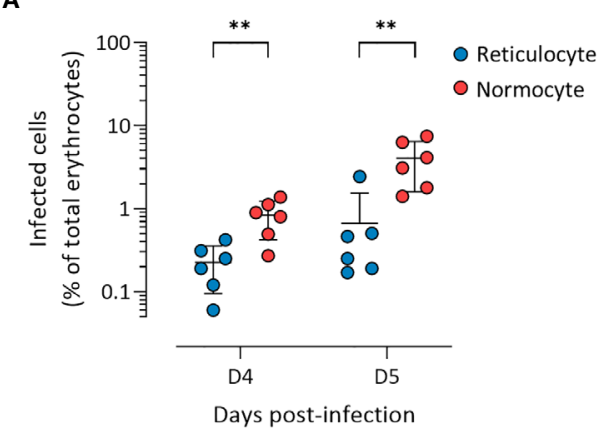

B

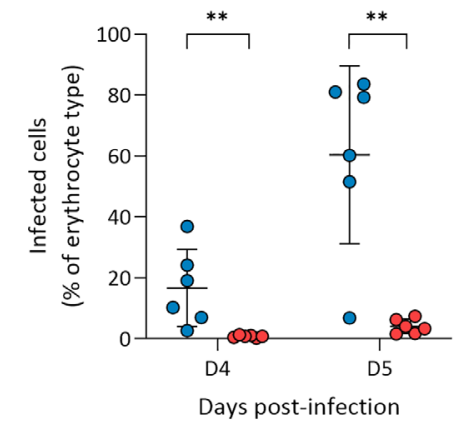

C

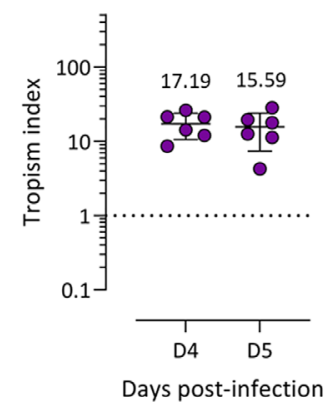

Py1.1

D

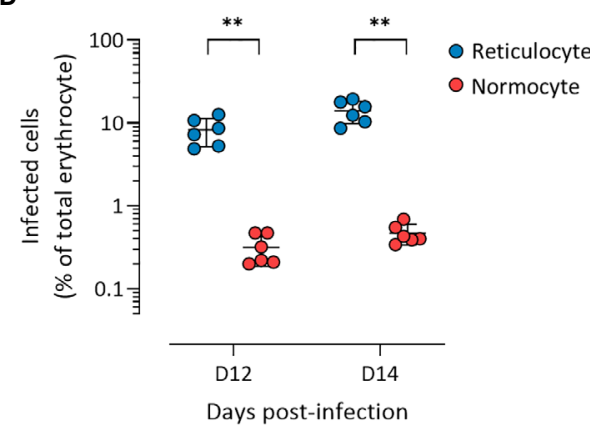

E

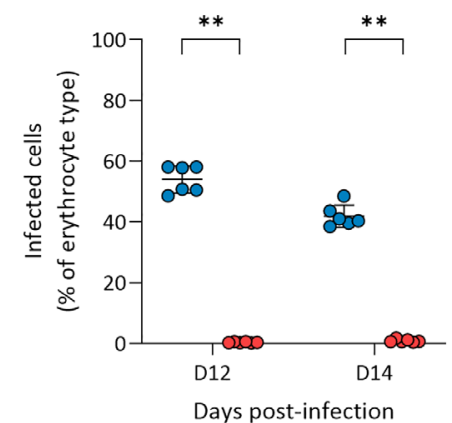

$\mathbf{F}$

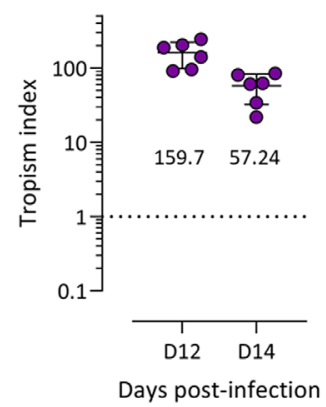

FIGURE $1 \mid P$. berghei ANKA (A-C) and $P$. yoelii 17X1.1 (D-F) prefer to invade reticulocytes in vivo. Flow cytometry data of peripheral blood taken from infected mice before the peak of infection (two days shown). Reticulocytes (blue circles) and normocytes (red circles) are defined as CD71+ CD98+ cells and CD71- CD98cells, respectively. Infected cells are Hoechst-positive. (A, D) Data shown are infected reticulocytes and infected normocytes, as a percentage of total erythrocytes. (B, E) Data normalized to the relative frequencies of reticulocytes and normocytes, by expressing infected reticulocytes and normocytes as a percentage of total reticulocytes and total normocytes, respectively. (C, F) The in vivo tropism index is calculated as the ratio of percentage of infected reticulocytes (out of total reticulocytes) to percentage of infected normocytes (out of total normocytes). The mean tropism indices ( $n=6$ mice) are shown. Dotted lines at tropism index $=1$ represent no erythrocyte preference. All error bars are standard deviations. ${ }^{\star \star} p<0.01$.

encounter and invade normocytes, therefore skewing the erythrocyte tropism. One way to normalize the different frequencies of reticulocytes and normocytes available is to compare the proportion of infected reticulocytes (out of total reticulocytes) with the proportion of infected normocytes (out of total normocytes). Using this approach, it is clear that $\mathrm{PbA}$ prefers to invade reticulocytes (Figure 1B). To further enable tropism comparisons across different strains, we also calculated the tropism index, which is a measure of how much more the parasite prefers reticulocytes over normocytes (see Materials and Methods). $\mathrm{PbA}$ had an in vivo tropism index of 15.6 (standard deviation, s.d. $=8.2$ ) at 5 days post-infection (dpi), i.e. $\mathrm{PbA}$ prefers to invade reticulocytes 16 times more than normocytes (Figure 1C).

Py1.1-infected mice harbored more infected reticulocytes than infected normocytes before the peak of parasitemia (Figure 1D), and the proportion of infected reticulocytes were higher than the proportion of infected normocytes (Figure 1E). During this period, the number of circulating reticulocytes increased substantially due to the anemia-induced reticulocytosis 
(Supplementary Figure S3), therefore merozoites encounter reticulocytes and normocytes at almost similar frequencies. The tropism index of Py1.1 was more than 50 at 14 dpi (Figure 1F), indicating that its preference for reticulocytes is stronger than that of $\mathrm{PbA}$

We observed more PccAS-infected normocytes than infected reticulocytes in circulation (Figure 2A), but when the erythrocyte frequencies were normalized as previously described, PccAS did not show a significant preference for either erythrocyte type (Figure 2B). Its in vivo tropism index was $0.9($ s.d. $=0.1)$ at
9 dpi, which indicates a mixed tropism (Figure 2C). For PvvS67, both types of measures showed significantly higher invasion into normocytes than reticulocytes (Figures 2D, E). PvvS67's tropism index is 0.5 (s.d. $=0.1$ ) at 9 dpi, i.e. its preference for reticulocytes is half of its normocyte preference (Figure 2F). Altogether, our in vivo tropism studies verified that $\mathrm{PbA}$ and $\mathrm{Py} 1.1$ prefer reticulocytes, PccAS has no erythrocyte preference, and PvvS67 has a slight normocyte tropism. We were also able to compare the extent of reticulocyte preference across strains, and it is clear that $\mathrm{Py} 1.1$ has a higher reticulocyte preference than $\mathrm{PbA}$ in vivo.
PccAS

A

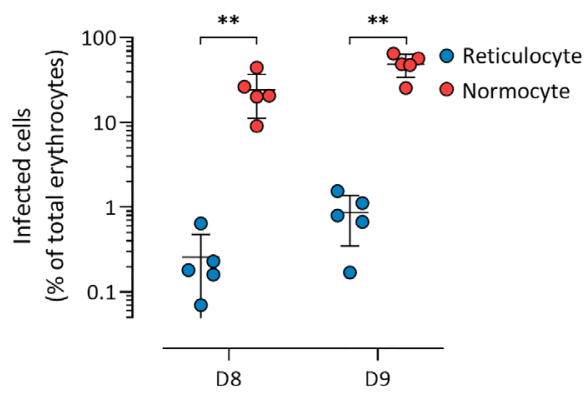

B

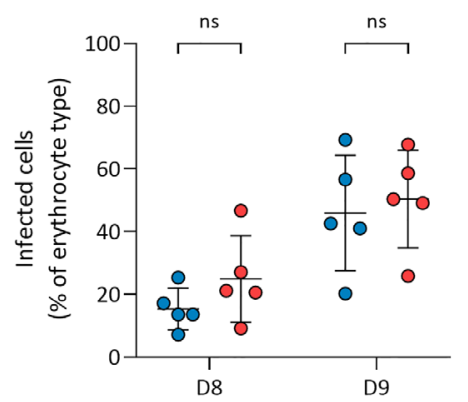

C

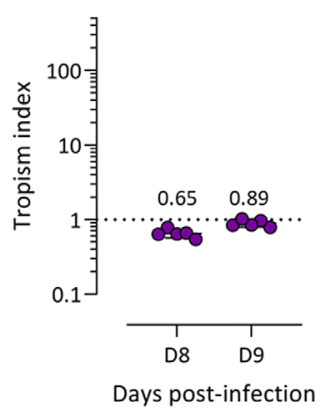

Pvv567

D

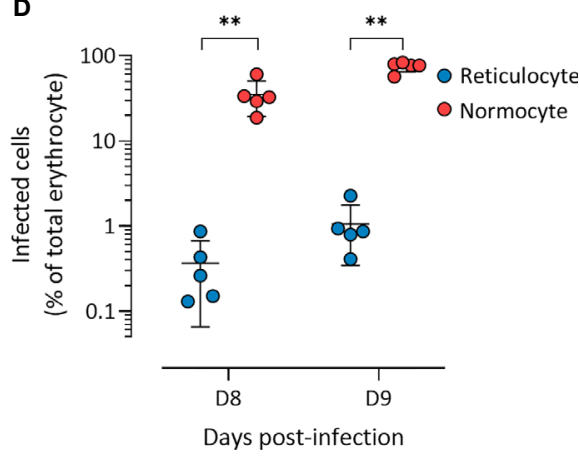

E

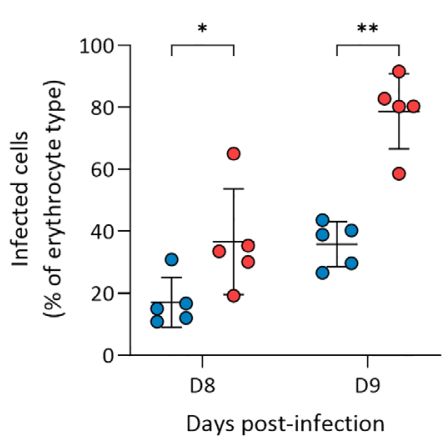

$\mathbf{F}$

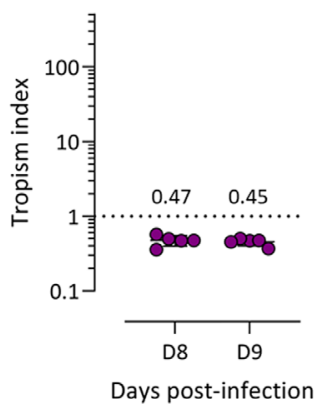

FIGURE $2 \mid$ P. chabaudi chabaudi AS has no erythrocyte preference (A-C) and P. vinckei vinckei S67 prefers normocytes (D-F) in vivo. Flow cytometry data of peripheral blood taken from infected mice before the peak of infection (two days shown). Reticulocytes (blue circles) and normocytes (red circles) are defined as CD71 + CD98+ cells and CD71- CD98- cells, respectively. Infected cells are Hoechst-positive. (A, D) Data shown are infected reticulocytes and infected normocytes, as a percentage of total erythrocytes. (B, E) Data normalized to the relative frequencies of reticulocytes and normocytes, by expressing infected reticulocytes and normocytes as a percentage of total reticulocytes and total normocytes, respectively. (C, $\mathbf{F})$ The in vivo tropism index is calculated as the ratio of percentage of infected reticulocytes (out of total reticulocytes) to percentage of infected normocytes (out of total normocytes). The mean tropism indices ( $n=5$ mice) are shown. Dotted lines at tropism index $=1$ represent no erythrocyte preference. All error bars are standard deviations. ns, not significant; ${ }^{*} p<0.05 ;{ }^{* *} p<0.01$. 


\section{Development of an Ex Vivo Tropism Assay}

We hypothesized that in vivo factors mentioned previously could skew tropism measurements, so we developed an ex vivo tropism assay to measure erythrocyte tropism in a controlled environment (Figure 3A). Late-stage parasites were incubated with enriched reticulocytes and normocytes, then new invasions into each cell type were measured by flow cytometry. To enrich mouse reticulocytes, mice were subjected to a phlebotomy routine to induce reticulocytosis and increase the number of circulating CD71+ CD98+ reticulocytes. Blood from these mice was further enriched for reticulocytes via Percoll density centrifugation. This two-step enrichment method consistently yielded $\sim 95 \%$ reticulocyte purity (Figure 3B) and most of these reticulocytes were CD71+ CD98+ (Supplementary Figure S4). Importantly, this allowed the output of the assay to be directly compared with our in vivo data, in which we also defined reticulocytes as CD71+ CD98+ erythrocytes. Normocytes were similarly enriched from blood of normal (untreated and uninfected) mice and most of these cells were CD71- CD98(Supplementary Figure S4).

The enriched reticulocytes and normocytes were then stained with different fluorescent cell dyes, CellTracker Deep Red (CTDR) and CellTrace Oregon Green (CTOG) respectively, so that they are distinguishable by flow cytometry (Figure 3A). To enrich late-stage parasites, blood from malaria-infected mice was subjected to magnet-activated cell sorting (MACS). MACS was able to efficiently enrich the hemozoin-containing late-stage parasites for all tested rodent malaria strains (Figure 3C). Finally, the parasites were incubated overnight with the target cells (stained reticulocytes and normocytes), and new invasions into each cell type were quantified by flow cytometry (Figure 3D). The CD71+ CD98+ reticulocytes did not mature considerably during incubation (Supplementary Figure S5), therefore we can be certain that newly-invaded cells in that population were reticulocytes during the point of merozoite invasion. Additionally, when the cell types were stained with the alternate fluorescent dyes, no significant difference in invasion was observed (Supplementary Figure S6), ruling out the possibility that the dyes were differentially affecting invasion into a particular cell type.

\section{Ex Vivo Tropism of Rodent Malaria at Equal Proportions of Reticulocytes and Normocytes}

The ratio of reticulocytes to normocytes in the tropism assay can be freely manipulated, therefore allowing us to measure the intrinsic erythrocyte preference of the parasite strains in an environment where reticulocytes and normocytes are equally available and not limited. At 50:50 reticulocyte:normocyte ratio, the reticulocyte invasion efficiencies of $\mathrm{PbA}$ and $\mathrm{Py} 1.1$ were significantly higher than normocyte invasion (Figure 4A). The assay also revealed no differences in reticulocyte and normocyte invasion for PccAS and PvvS67.

Considering the fact that PccAS and PvvS67 showed no erythrocyte preference ex vivo, we can define the baseline of reticulocyte preference at tropism index $=1.5$ (1.5 times higher reticulocyte preference) and the baseline of normocyte preference at tropism index $=0.67$ (1.5 times higher normocyte preference) (Figure 4B). With tropism indices of more than 1.5, both $\mathrm{PbA}$ and Py1.1 showed a marked tropism for reticulocytes. PbA was 5.2 (s.d. $=1.1)$ times more likely to invade a reticulocyte than a normocyte, and Py1.1 preferred reticulocytes 2.5 (s.d. $=0.4$ ) times more than normocytes. In contrast to our in vivo data, $\mathrm{PbA}$ displayed a stronger preference for reticulocytes compared to Py1.1. Additionally, the tropism indices of $\mathrm{PbA}$ and Py1.1 were also much lower ex vivo. Overall, these results show that in an environment with equal availabilities of target erythrocytes, $\mathrm{PbA}$ and Py1.1 have an intrinsic preference for reticulocytes, whereas PccAS and PvvS67 can invade both erythrocyte types equally well.

\section{Ex Vivo Tropism of Rodent Malaria at Variable Proportions of Reticulocytes and Normocytes}

Due to some disparity between in vivo and ex vivo observations, we next investigated whether target cell availability directly affects erythrocyte tropism. During the in vivo timepoints, normocytes represent a wide majority (>90\%) of circulating erythrocytes for all parasite strains except Py1.1 (Supplementary Figure S3). Even though we tried to account for the vast difference in reticulocyte and normocyte availabilities by normalizing invasion to the respective cell frequencies, we cannot rule out that the excessive abundance of normocytes might be directly affecting invasion into a particular cell type. To simulate a wider range of erythrocyte frequencies, we varied the proportions of erythrocytes added to the tropism assay (10:90, 30:70, 50:50, 70:30, and 90:10 reticulocyte:normocyte ratio) and compared the invasion efficiencies.

For $\mathrm{PbA}$, there were more invaded reticulocytes than invaded normocytes at every ratio except when reticulocytes only constituted $10 \%$ of target cells (Figure 5A). However, when the invasion efficiencies were normalized to the corresponding cell population frequencies (see Materials and Methods), reticulocyte invasion was higher at every ratio (Figure 5B). This indicates that the parasite's reticulocyte preference is intrinsic and unaffected by the surrounding erythrocyte composition. Reflecting this, PbA's tropism index was stable across the different ratios (Figure 5C). Additionally, this shows that the tropism index is a reliable indicator of a parasite's intrinsic erythrocyte preference. The results for Py1.1 are mostly similar to $\mathrm{PbA}$, in which there were more infected normocytes only at the minimum reticulocyte frequency (Figure 5D). This superficial normocyte tropism was abolished once the relative erythrocyte frequencies were accounted for (Figure 5E). The tropism index of Pyl.1 also remained constant at around 2 (Figure 5F).

The mixed tropism of PccAS was clearly seen at all erythrocyte ratios; an increase in reticulocyte invasion was accompanied by a decrease in normocyte invasion as the reticulocyte frequency increased, and the two invasion lines intersected around the 50:50 ratio (Figure 6A). Interestingly, the total invasion (sum of reticulocyte and normocyte invasions) decreased at higher reticulocyte frequencies, indicating that PccAS does not invade as well (regardless of target cell type) in a reticulocyte-rich environment. The mixed tropism of PccAS was stable at different ratios, as shown by the minimal difference 
A

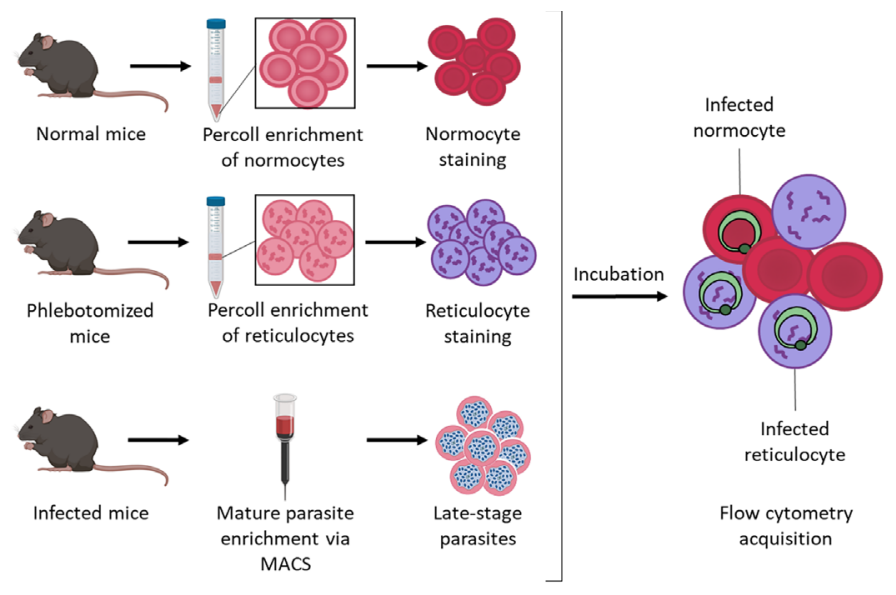

B

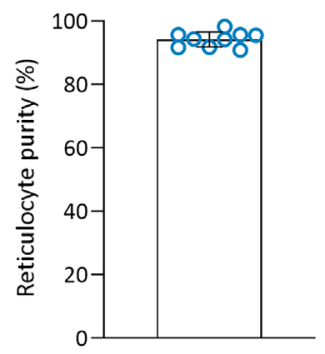

C
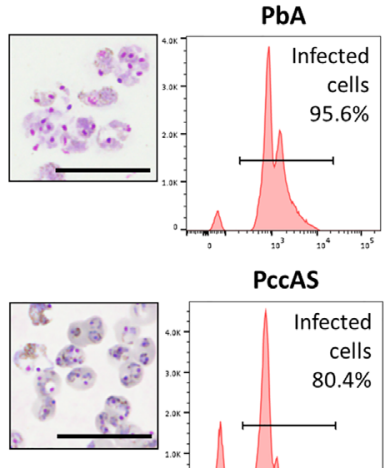

D

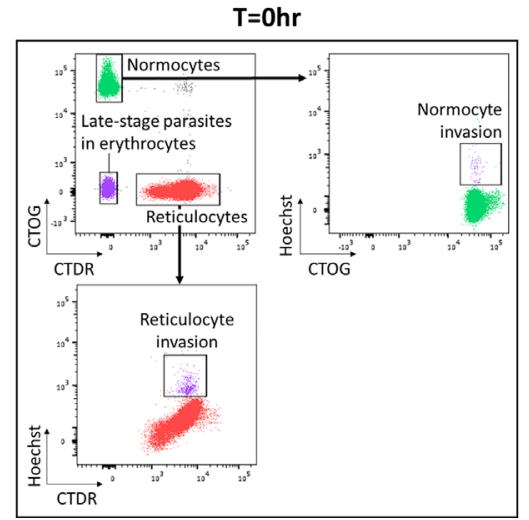

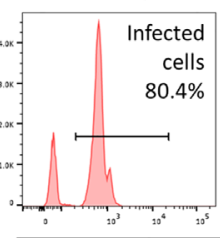

Hoechst
Py1.1

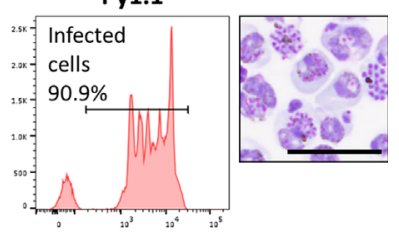

PvvS67

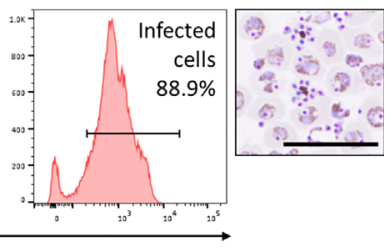

$\mathrm{T}=12 \mathrm{hr}$

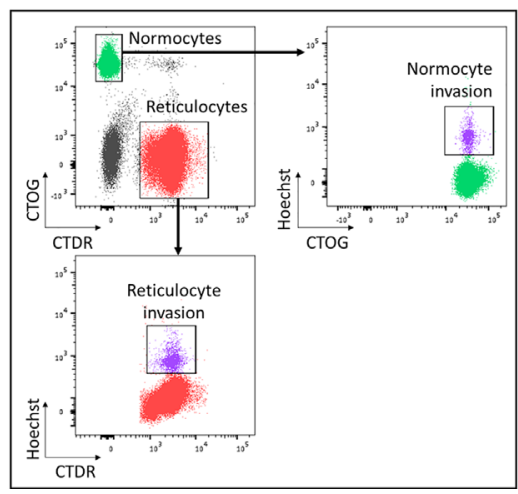

FIGURE 3 | Development of an ex vivo tropism assay to measure erythrocyte preference of rodent malaria. (A) Schematic showing workflow of the assay. Normocytes are enriched by Percoll density centrifugation of blood taken from normal mice (untreated and uninfected). Reticulocytes are enriched similarly from blood taken from mice that underwent a phlebotomy routine. Enriched cells are then stained with different fluorescent dyes. Late-stage parasites are enriched from infected blood via magnetic-activated cell sorting (MACS). The cells are then incubated together for 12 hrs and measured by flow cytometry. Images were created on Biorender.com. (B) Reticulocyte purity (based on thiazole orange staining) after the two-step reticulocyte enrichment. Data from 9 independent experiments. Error bars represent standard deviation. (C) Late-stage parasite purity post-MACS for the four major rodent malaria strains. Histogram showing Hoechst levels and Giemsa-stained thin smears of enriched cells. Most of the enriched cells consist of late-stage parasites. Scale bar of microscopy images $=20 \mu \mathrm{m}$. PbA, $P$. berghei ANKA. Py1.1, P. yoelii 17X1.1. PccAS, P. chabaudi chabaudi AS. PvvS67, P. vinckei vinckei S67. (D) Flow cytometry gating strategy. At T=0hr, reticulocytes stained with CellTracker Deep Red (CTDR) are easily distinguishable from normocytes stained with CellTrace Oregon Green (CTOG). Late-stage parasites within erythrocytes are negative for both dyes. At T=12hr, new invasions into reticulocytes and normocytes can be detected by Hoechst. 

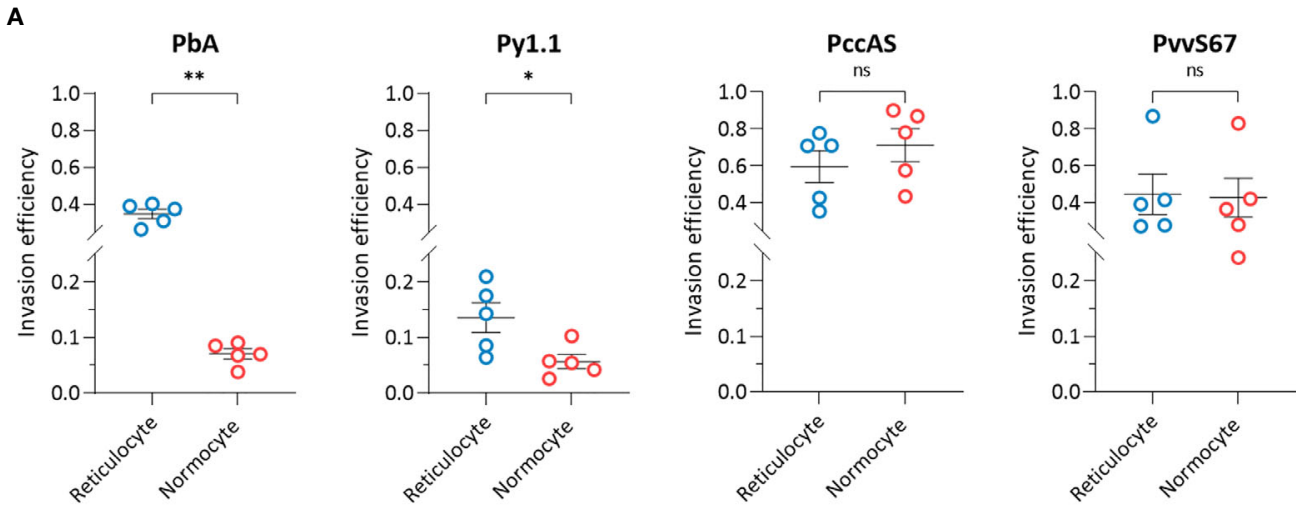

B

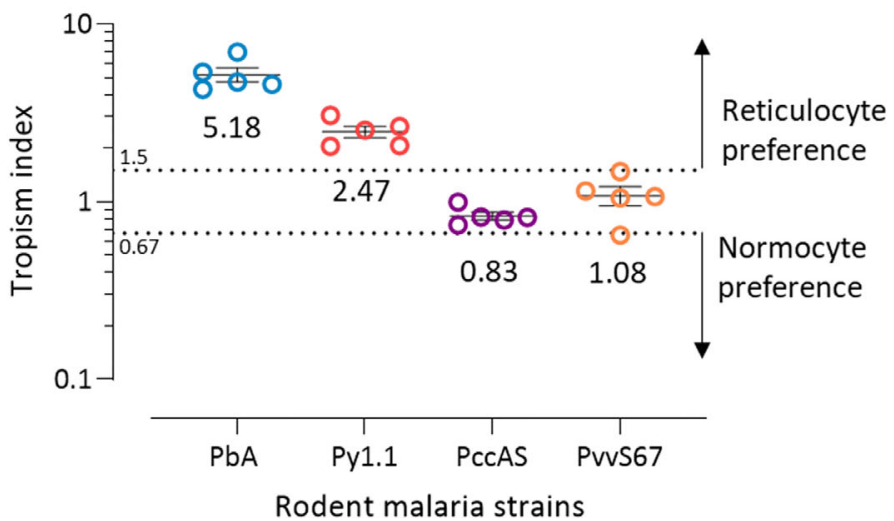

FIGURE 4 | Ex vivo tropism of rodent malaria strains at equal reticulocyte:normocyte ratio. (A) Reticulocyte and normocytes invasion efficiencies (see Materials and Methods for calculation) were compared for all strains to determine erythrocyte preference. Data shown for each of the parasite strains are from 5 independent experiments. Error bars represent standard error of mean (SEM). ns, not significant; ${ }^{*} p<0.05$; ${ }^{* *} p<0.01$. (B) Tropism indices are derived from the invasion efficiencies in (A). Tropism index was calculated as the ratio of reticulocyte invasion efficiency to normocyte invasion efficiency. Dotted lines at tropism index $=0.67$ and 1.5 represent the boundaries of mixed tropism, as determined by the spread of PccAS's and Pv S67's data. Tropism indices of $>1.5$ indicate a preference for reticulocytes and tropism indices of $<0.67$ indicate normocyte tropism. The mean tropism indices are shown, and error bars represent $\mathrm{SEM}$. PbA, $P$. berghei ANKA Py1.1, P. yoelii 17X1.1. PccAS, P. chabaudi chabaudi AS. PvvS67, P. vinckei vinckei S67.

between normalized invasion efficiencies (Figure 6B), and as shown by the tropism index (Figure 6C). PvvS67 also displayed similar trends to PccAS: the intersection of invasion lines near the 50:50 ratio (Figure 6D), the normalized invasion of both cell types were similar to one another at most ratios (Figure 6E), and the tropism index was mostly unchanged (Figure 6F). The total invasion of PvvS67 also decreased drastically at high reticulocyte numbers (Figure 6D). This was not seen in the reticulocytepreferring strains, $\mathrm{PbA}$ (Figure 5A) and Pyl.1 (Figure 5D). Taken together, these findings support the idea that erythrocyte preference of rodent malaria is intrinsic in nature and unaffected by the availability of target erythrocytes.

\section{DISCUSSION}

The surface proteome of reticulocytes and normocytes are remarkably different due to protein downregulation during reticulocyte maturation in humans (Wilson et al., 2016; Chu et al., 2018; Gautier et al., 2018) and mice (Gautier et al., 2020). Considering the fact that erythrocytic invasion by malaria parasites is mediated by erythrocyte receptor-parasite ligand interactions, the erythrocyte tropism of the parasites could hint at the key receptors necessary for invasion. However, when we attempted to classify rodent malaria species based on their erythrocyte tropism, we realized that previous tropism studies are inconsistent from one another in terms of measuring and evaluating tropism. This made cross-species comparisons difficult and there was no way to estimate their relative preferences for reticulocytes and normocytes.

Therefore, using flow cytometry to objectively define the erythrocyte populations, we performed comprehensive in vivo tropism measurements for the major rodent malaria strains. Our results broadly agree with previous observations: $\mathrm{PbA}$ and $\mathrm{Py} 1.1$ have a strong reticulocyte preference (Fahey and Spitalny, 1984; Deharo et al., 1996; Cromer et al., 2006; Otsuki et al., 2009; 
PbA

A

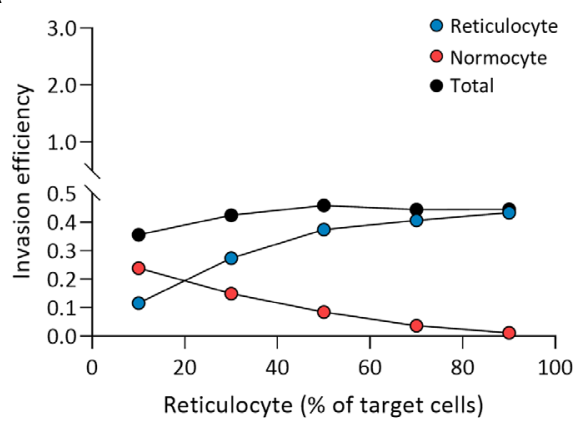

B

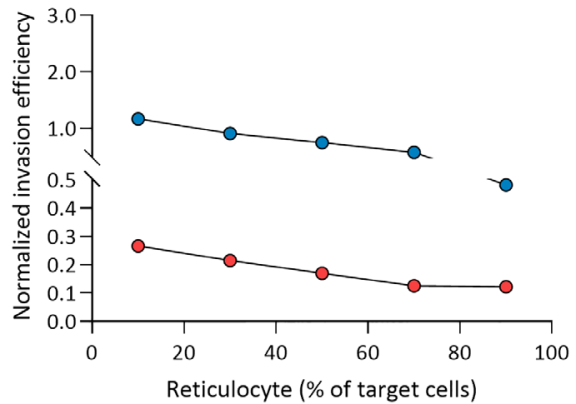

C

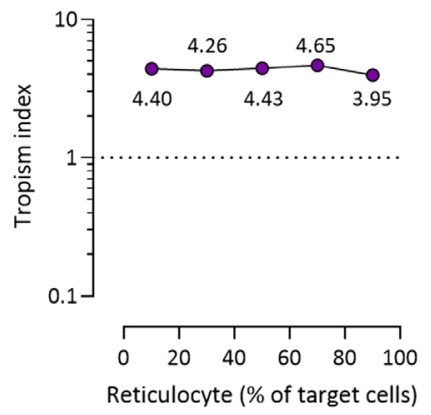

Py1.1

D

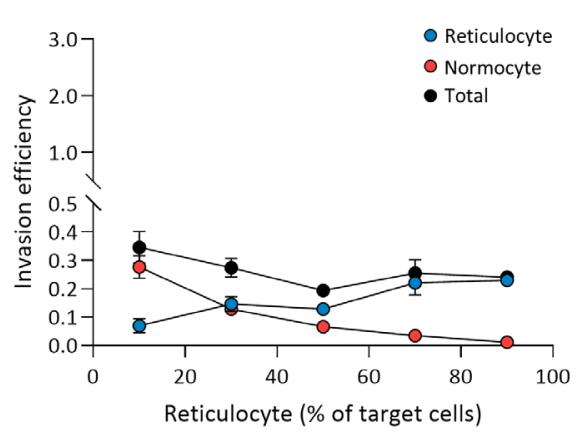

E

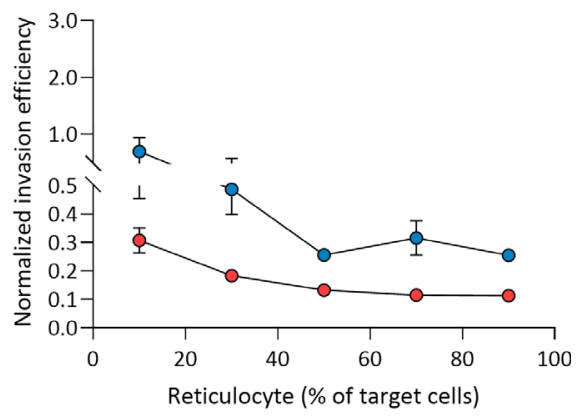

F

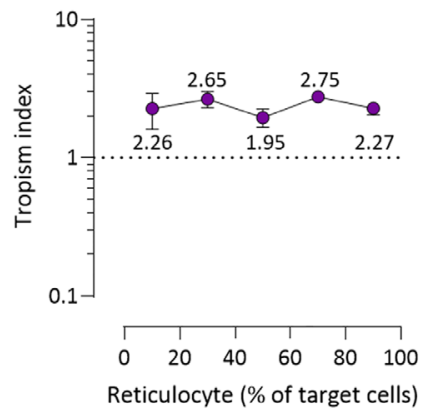

FIGURE 5 | Ex vivo tropism of $P$. berghei ANKA (A-C) and P. yoelii 17X1.1 (D-F) at variable reticulocyte:normocyte ratios. (A, D) Reticulocyte (blue) and normocyte (red) invasion efficiencies (see Materials and Methods for calculation) were compared at five different erythrocyte ratios, 10:90, 30:70, 50:50, 70:30, 90:10 reticulocyte:normocyte. Total invasion efficiency (black) is the sum of reticulocyte and normocyte invasions. (B, E) Reticulocyte and normocyte invasion efficiencies were normalized to their respective population frequencies (see Materials and Methods). (C, F) Tropism indices were calculated from the ratio of normalized reticulocyte invasion efficiency to the normalized normocyte invasion efficiency. Mean tropism indices are also shown. Dotted lines at tropism index $=1$ represent mixed tropism. All error bars represent standard deviation. $n=5$ technical replicates, for each strain.

Martín-Jaular et al., 2013; Thakre et al., 2018), PccAS has a mixed tropism (Jarra and Brown, 1989), and PvvS67 has a slight normocyte tropism (Viens et al., 1971; Vigário et al., 2001). An important distinction between the present study and those before is that we only strictly compared normocytes with the earliest stages of reticulocytes (which still retain many surface proteins). This keeps the two erythrocyte populations well-defined and homogenous, allowing for more accurate measurements. The differential expression of surface proteins by the two populations also paves way to future mechanistic studies investigating the receptors mediating tropism. More importantly, our approach is able to rank the different rodent malaria strains based on their erythrocyte preference in vivo: Py1.1 (strongest reticulocyte preference) $>$ PbA $>$ PccAS > PvvS67 (strongest normocyte preference).

It is important to realize that the mere comparison of proportion of infected reticulocytes with the proportion of infected normocytes is not adequate in determining the true erythrocyte preference in vivo. Cromer et al. (2006) also realized this, and they attributed the problem to the dynamics of the infection; the changing rates of reticulocyte production and erythrocyte destruction could affect in vivo tropism 
A
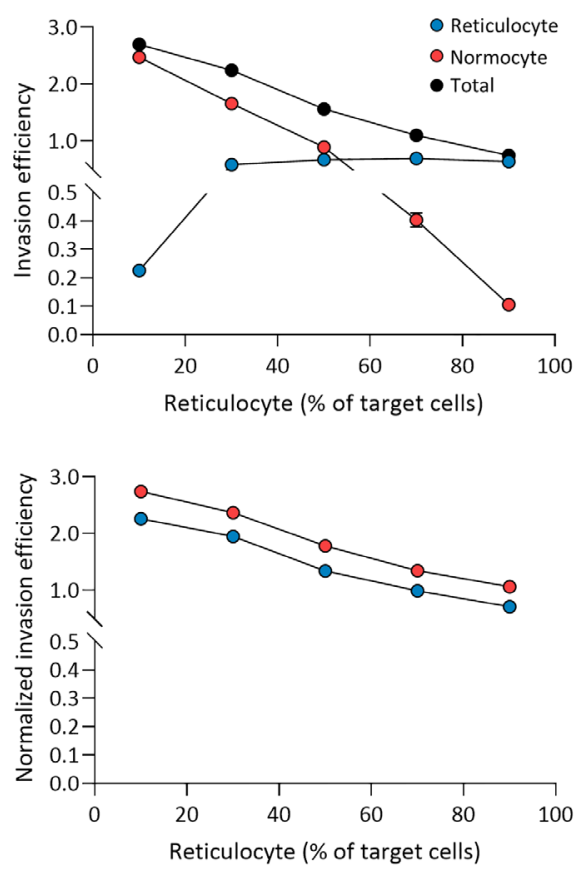

C

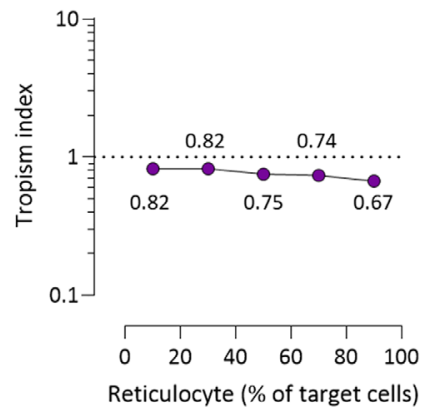

PvvS67

D

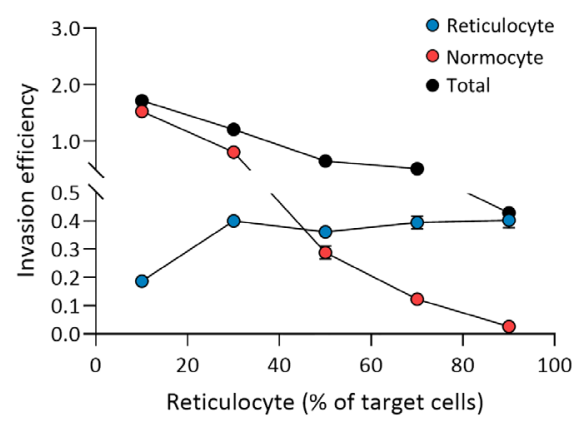

$\mathbf{E}$

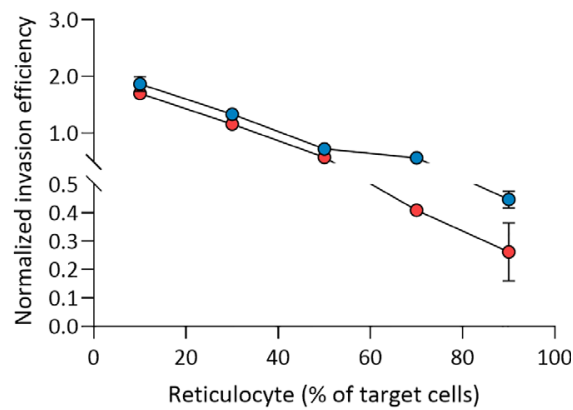

$\mathbf{F}$

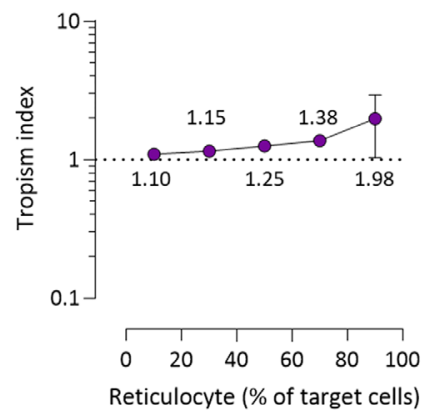

FIGURE 6 | Ex vivo tropism of $P$. chabaudi chabaudi AS (A-C) and P. vinckei vinckei S67 (D-F) at variable reticulocyte:normocyte ratios. (A, D) Reticulocyte (blue) and normocyte (red) invasion efficiencies (see Materials and Methods for calculation) were compared at five different erythrocyte ratios, 10:90, 30:70, 50:50, 70:30, 90:10 reticulocyte:normocyte. Total invasion efficiency (black) is the sum of reticulocyte and normocyte invasions. (B, E) Reticulocyte and normocyte invasion efficiencies were normalized to their respective population frequencies (see Materials and Methods). (C, F) Tropism indices were calculated from the ratio of normalized reticulocyte invasion efficiency to the normalized normocyte invasion efficiency. Mean tropism indices are also shown. Dotted lines at tropism index $=1$ represent mixed tropism. All error bars represent standard deviation. $n=5$ technical replicates, for each strain.

measurements. They developed a mathematical model to account for this and concluded that $P$. berghei prefers reticulocytes $\sim 150$ times over normocytes.

Here we circumvented the problems of infection dynamics and other potential in vivo confounding factors (like parasite sequestration and preferential removal of infected reticulocytes) by developing an ex vivo tropism assay. In an environment where reticulocytes and normocytes were equally available, we observed that $\mathrm{PbA}$ and Py1.1 prefer reticulocytes (5 and 2.5 times more than normocytes, respectively), whereas PccAS and PvvS7 showed no inclination for either erythrocyte population. The most striking observation from this is that PbA's and Pyl.1's reticulocyte preference ex vivo was much lower than our in vivo estimates (and the estimates in Cromer et al., 2006). We postulate that this discrepancy is due to the parasite's capability to invade cells and replicate within hematopoietic sites of the host (e.g. bone marrow, spleen and liver), as supported by a growing number of evidence [reviewed in (Venugopal et al., 2020)] for P. falciparum (Joice et al., 2014), P. vivax (Obaldia et al., 2018), and P. berghei (De Niz et al., 2018; Lee et al., 2018). P. berghei was shown to parasitize early reticulocytes in the extravascular compartments of the bone 
marrow and spleen (De Niz et al., 2018; Lee et al., 2018). P. yoelii was also found in the bone marrow of infected mice (Imai et al., 2013). If a significant portion of invasion takes place in these reticulocyte-rich hematopoietic niches before the infected cells enter the bloodstream, then this would artificially inflate the reticulocyte preference of our peripheral blood data.

The role of the hematopoietic niche as a major developmental site for blood-stage malaria also raises an intriguing link with erythrocyte tropism. Do reticulocyte-preferring strains develop in hematopoietic organs due to the abundance of reticulocytes there, or is the hematopoietic environment inherently beneficial for parasite development and as an indirect consequence, the parasites have adapted to invading the cells present there (which happens to be reticulocytes)? On the contrary, we also observed that PccAS and PvvS67, which do not have the propensity to invade reticulocytes, have reduced total invasion efficiencies in a reticulocyte-rich environment. It is likely that these strains primarily invade in the peripheral circulation, where reticulocytes are less abundant, as opposed to PbA and Py1.1.

Notably, none of the strains tested here showed a preference for normocytes, which is surprising considering that normocytes are easily accessible and constitute the bulk of circulating erythrocytes. This could be due to the possibility that many of the receptors used for normocyte invasion are also found in similar abundance (if not more) on reticulocytes. Another possible explanation is that it is directly beneficial for the parasite to invade reticulocytes due to the relatively (compared to normocytes) metabolic-rich environment of the cells. Reticulocytes still retain some metabolic pathways that can be capitalized by the parasites (Srivastava et al., 2015). Reticulocyte invasion could also be a parasite evasion strategy; reticulocytes express higher levels of the 'do not eat me' signal, CD47, allowing infected reticulocytes to escape phagocyte clearance (Banerjee et al., 2015).

To sum up our ex vivo findings, $\mathrm{PbA}$ showed the strongest preference for reticulocytes, followed by Py1.1, whereas both PccAS and PvvS67 showed mixed tropism. Since the assay is devoid of external factors that could affect tropism, we believe that $\mathrm{PbA}$ 's and Py1.1's strong reticulocyte preference is indicative of the erythrocyte receptors that are essential for their invasion. These unidentified receptors could be more abundant on the surface of reticulocytes than normocytes, allowing a higher probability of successful invasion into reticulocytes. Another possibility is that these receptors are only found on reticulocytes, but the parasites are also able to utilize lesspreferred receptors on normocytes. A wide range of host receptors have been identified for $P$. falciparum invasion (Cowman et al., 2017), but none of them explain its tropism (prefers reticulocytes but can also invade normocytes). We suggest that $\mathrm{PbA}$ and $\mathrm{Py} 1.1$ are good mouse models to study $P$. falciparum invasion, considering that they show similar tropism signatures. Even though none of the rodent malaria strains tested here (and by others) showed a strict tropism to reticulocytes like $P$. vivax, reticulocyte-preferring strains still remain potential models to identify new reticulocyte-specific receptors. Our work here hopefully encourages deeper investigations into determining the receptor-ligand interactions mediating tropism, and identifying interventions that inhibit these interactions, consequently halting the reinvasion of erythrocytes.

\section{MATERIALS AND METHODS}

\section{Mice}

Male and female C57BL/6J mice (aged 5-9 weeks old) were used. For reticulocyte and normocyte enrichment, mice were age- and gender-matched. Mice were housed under specific pathogen-free (SPF) conditions in the $\mathrm{A}^{\star} \mathrm{STAR}$ Biomedical Resource Centre (Biopolis, Singapore). All animal procedures were approved by the $A^{*}$ STAR Biomedical Resource Centre Institutional Animal Care and Use Committee (IACUC \#181314) in accordance with guidelines set by the Animal \& Veterinary Service (AVS) and National Advisory Committee for Laboratory Animal Research (NACLAR) of Singapore.

\section{Parasite Strains and Mice Infection}

The rodent malaria strains used were Plasmodium berghei ANKA clone 15cy1 (PbA) (Janse et al., 2006), P. yoelii 17X clone 1.1 (Py1.1) (Weiss et al., 1989), P. chabaudi chabaudi clone AS (PccAS) (Cornelissen et al., 1985) and P. vinckei vinckei S67 (PvvS67) (Bafort, 1967). The origin of the different parasite strains was ascertained using a combination of 19 microsatellite probes described elsewhere (Li et al., 2007). Parasite stocks were made in Alsever's buffer and stored in liquid nitrogen. Mice were infected intraperitoneally (i.p.) with $10^{6}$ infected erythrocytes from the parasite stocks.

\section{In Vivo Tropism Follow-Up via Flow Cytometry}

$1 \mu \mathrm{L}$ tail blood of infected mice was collected into $100 \mu \mathrm{L}$ phosphate-buffered saline (PBS). Samples were stained with Hoechst 33342 (at $8 \mu \mathrm{M}$; Sigma \#B2261), rat IgG2b anti-mouse CD45-PE/Cy7 (at $2 \mu \mathrm{g} / \mathrm{mL}$; BD \#552848), rat IgG2a anti-mouse CD71-APC (at $2 \mu \mathrm{g} / \mathrm{mL}$; Invitrogen \#17-0711-82), and rat IgG2a anti-mouse CD98-PE (at $2 \mu \mathrm{g} / \mathrm{mL}$; Invitrogen \#12-0981-83) for 15-20 mins. Cells were washed with PBS and immediately acquired on the BD LSRII flow cytometer, with a minimum of 100,000 cells recorded. Data were analyzed using FlowJo software (version 10). Erythrocytes were gated as CD45- cells. Tropism index for in vivo experiments was calculated as the ratio of the proportion of CD71+CD98+ reticulocytes that were infected, to the proportion of CD71-CD98- normocytes that were infected.

\section{Late-Stage Parasite Enrichment}

Blood from infected mice was collected into heparinized parasite media (RPMI $1640+20 \%$ heat-inactivated fetal bovine serum (FBS) $+40 \mu \mathrm{g} / \mathrm{mL}$ gentamicin). Leukocytes were depleted by passing the blood suspension through non-woven fabric (NWF) filter twice (Tao et al., 2011), and washed with parasite media. Late-stage parasites of all rodent malaria strains were enriched via magnet-activated cell sorting (MACS). Infected blood was 
passed through an LD column (Miltenyi Biotec \#130-042-901) attached to a magnet. Late-stage parasites, which were retained in the column, were flushed out with parasite media. The purity of late-stage parasites was assessed with Hoechst staining and Giemsa-stained thin smears. Among the late-stage parasites, trophozoites and schizonts represent the majority stages for all parasite strains (>95\%) (Supplementary Figure S7). Although there were some uninfected erythrocytes present in the late-stage parasite-enriched fraction, these cells only make up $<2 \%$ of total cells after the addition of target erythrocytes, and therefore unlikely to affect the invasion of target cells.

\section{Reticulocyte and Normocyte Enrichment}

Mice were subjected to an adapted phlebotomy routine to induce reticulocytosis (Liu et al., 2010). On Days -4 and -2, isofluraneanesthetized mice were bled $600 \mu \mathrm{L}$ via the retro-orbital route and injected with $1 \mathrm{~mL}$ PBS i.p. to replace the loss of fluid. On Day 0 , the mice were exsanguinated under anesthesia into heparinized parasite media. The reticulocyte-rich blood suspension was leukodepleted and washed as previously, then resuspended to $50 \%$ hematocrit with parasite media. To enrich reticulocytes by Percoll density centrifugation, $2 \mathrm{~mL} 45 \%$ isotonic Percoll was overlaid on $3 \mathrm{~mL} 70 \%$ isotonic Percoll, and $1 \mathrm{~mL}$ of the blood suspension was laid on the $45 \%$ layer. The blood was then centrifuged at $250 \times \mathrm{g}$ for $30 \mathrm{mins}$ at room temperature. The reticulocyte band, which can be found at the interface of the two Percoll layers, was removed carefully and washed with parasite media. To enrich normocytes, normal mice were bled and the blood was processed similarly to above. After Percoll centrifugation, normocytes were collected from the pellet. The purity of enriched reticulocytes and normocytes were assessed by staining with thiazole orange (at $0.5 \mu \mathrm{g} / \mathrm{mL}$; Sigma \#390062), $1 \mu \mathrm{g} / \mathrm{mL}$ anti-mouse CD71-APC and $1 \mu \mathrm{g} / \mathrm{mL}$ antimouse CD98-PE for 15-20 mins.

\section{Ex Vivo Tropism Assay}

Enriched reticulocytes and normocytes were resuspended to 1\% hematocrit with PBS and stained with CellTracker Deep Red (at 2 $\mu \mathrm{M}$; Invitrogen \#C34565) and CellTrace Oregon Green (at $15 \mu \mathrm{M}$; Invitrogen \#C34555), respectively. After 30 mins of staining at $37^{\circ} \mathrm{C}$, warm FBS was added to stop the staining reaction. Cells were then washed twice with media, with another 10 mins incubation during the second wash. Stained reticulocytes and normocytes were mixed to the preferred ratios (10:90, 30:70, $50: 50,70: 30$, or $90: 10$ ) and resuspended in parasite media to $2 \%$ hematocrit. Enriched late-stage parasites were then added at around $5-10 \%$ final parasitemia and the cells were incubated at $37^{\circ} \mathrm{C}$ for 12 hours. At Time $=0 \mathrm{hr}$ (i.e. after the addition of parasites), aliquots of samples were mixed with PBS and stained with $8 \mu \mathrm{M}$ Hoechst for 15-20 mins. $300 \mu \mathrm{L}$ PBS was then added to quench the reaction and the samples were immediately acquired. The staining and flow cytometry acquisition was repeated at Time = $12 \mathrm{hr}$. This 12-hour incubation allows sufficient time for the trophozoites and schizonts to develop and release merozoites, and yet not too long until a second round of invasion occurs [e.g. the asexual cycle of different $P$. yoelii strains vary between 18-24 hours
(Killick-Kendrick and Warben, 1968; Gautret et al., 1994)] or for the target reticulocytes to mature into normocytes.

\section{Invasion Efficiency Calculation and Normalization}

Invasion efficiency $=\left[\frac{(T 12 h \text { invasion }-T 0 h \text { invasion })}{T 0 h \text { Late stage parasites }}\right] \times 0.5$

The equation above shows how invasion efficiencies of reticulocytes and normocytes were calculated for the 50:50 reticulocyte:normocyte ratio experiments. The values for 'T0h reticulocyte invasion', 'T12h reticulocyte invasion', 'T0h normocyte invasion', 'T12h normocyte invasion', and 'T0h Latestage parasites' were directly taken from the flow cytometry gates (see Figure 3D). The resultant value (in squared brackets) is then multiplied by 0.5 because reticulocytes and normocytes each represent half of the target cell population. For the variable ratios experiments, instead of 0.5 , the resultant value is multiplied accordingly. For example, to determine the reticulocyte invasion efficiency when reticulocytes consist $90 \%$ of target cells, the resultant value is multiplied by 0.9 instead.

To normalize the invasion efficiency to the corresponding cell type frequency, the invasion efficiency is divided by the cell frequency. For example, to get the normalized reticulocyte invasion efficiency when reticulocytes consist $90 \%$ of target cells, the reticulocyte invasion efficiency is divided by 0.9 . Consequently, the normalized value is equivalent to the value in squared brackets.

\section{Statistical Analysis}

Statistical analyses were performed on GraphPad Prism software (version 9). All data were analyzed non-parametrically using Mann-Whitney test, and $p$-values $<0.05$ were considered statistically significant.

\section{DATA AVAILABILITY STATEMENT}

The raw data supporting the conclusions of this article will be made available by the authors, without undue reservation.

\section{ETHICS STATEMENT}

The animal study was reviewed and approved by $\mathrm{A}^{\star} \mathrm{STAR}$ Biomedical Resource Centre Institutional Animal Care and Use Committee (IACUC).

\section{AUTHOR CONTRIBUTIONS}

YL, LR, and BM conceptualized and designed the study. YL and EL performed the experiments. YL, EL, LR, and BM analyzed and interpreted the data. YL, LR, and BM prepared the manuscript. All authors contributed to the article and approved the submitted version. 


\section{FUNDING}

The project was supported by core funds given by the Agency for Science, Technology and Research (A*STAR) to the Singapore Immunology Network (SIgN) (to LR and BM) and $A^{\star}$ STAR ID Labs (to LR); and by the National University Health System (NUHS) Start-up grant (NUHSRO/2018/006/SU/01) (to BM). YL is funded by the Singapore International Graduate Award (SINGA) by $A^{\star S T A R}$. The funding sources had no role in the study design; collection, analysis and interpretation of data; in the writing of the report; and in the decision to submit the paper for publication.

\section{REFERENCES}

Bafort, J. (1967). La Transmission Cyclique Du Plasmodium Vinckei. Ann. Soc Belg. Med. Trop. 47, 271-276.

Banerjee, R., Khandelwal, S., Kozakai, Y., Sahu, B., and Kumar, S. (2015). CD47 Regulates the Phagocytic Clearance and Replication of the Plasmodium Yoelii Malaria Parasite. Proc. Natl. Acad. Sci. 112, 3062-3067. doi: 10.1073/ pnas. 1418144112

Chin-Yee, I., Keeney, M., and Lohmann, R. C. (1991). Flow Cytometric Reticulocyte Analysis Using Thiazole Orange; Clinical Experience and Technical Limitations. Clin. Lab. Haematol. 13, 177-188. doi: 10.1111/ j.1365-2257.1991.tb00267.x

Chu, T. T. T., Sinha, A., Malleret, B., Suwanarusk, R., Park, J. E., Naidu, R., et al. (2018). Quantitative Mass Spectrometry of Human Reticulocytes Reveal Proteome-Wide Modifications During Maturation. Br. J. Haematol. 180, 118-133. doi: 10.1111/bjh.14976

Claser, C., Malleret, B., Gun, S. Y., Wong, A. Y. W., Chang, Z. W., Teo, P., et al. (2011). Cd8+ T Cells and IFN- $\gamma$ Mediate the Time-Dependent Accumulation of Infected Red Blood Cells in Deep Organs During Experimental Cerebral Malaria. PloS One 6, e18720. doi: 10.1371/journal.pone.0018720

Cornelissen, A. W. C. A., Langsley, G., Walliker, D., and Scaife, J. G. (1985). Gametocytogenesis and Ribosomal rRNA Gene Organisation in the Rodent Malarias Plasmodium Chabaudi and Plasmodium Berghei. Mol. Biochem. Parasitol. 14, 165-174. doi: 10.1016/0166-6851(85)90035-0

Cowman, A. F., Tonkin, C. J., Tham, W.-H., and Duraisingh, M. T. (2017). The Molecular Basis of Erythrocyte Invasion by Malaria Parasites. Cell Host Microbe 22, 232-245. doi: 10.1016/j.chom.2017.07.003

Cromer, D., Evans, K. J., Schofield, L., and Davenport, M. P. (2006). Preferential Invasion of Reticulocytes During Late-Stage Plasmodium Berghei Infection Accounts for Reduced Circulating Reticulocyte Levels. Int. J. Parasitol. 36, 1389-1397. doi: 10.1016/j.ijpara.2006.07.009

Deharo, E., Coquelin, F., Chabaud, A. G., and Landau, I. (1996). The Erythrocytic Schizogony of Two Synchronized Strains of Plasmodium Berghei, NK65 and ANKA, in Normocytes and Reticulocytes. Parasitol. Res. 82, 178-182. doi: $10.1007 /$ s004360050091

De Niz, M., and Heussler, V. T. (2018). Rodent Malaria Models: Insights Into Human Disease and Parasite Biology. Curr. Opin. Microbiol. 46, 93-101. doi: 10.1016/j.mib.2018.09.003

De Niz, M., Meibalan, E., Mejia, P., Ma, S., Brancucci, N. M. B., Agop-Nersesian, C., et al. (2018). Plasmodium Gametocytes Display Homing and Vascular Transmigration in the Host Bone Marrow. Sci. Adv. 4, eaat3775. doi: 10.1126/ sciadv.aat 3775

Dertinger, S. D., Torous, D. K., Hall, N. E., Tometsko, C. R., and Gasiewicz, T. A. (2000). Malaria-Infected Erythrocytes Serve as Biological Standards to Ensure Reliable and Consistent Scoring of Micronucleated Erythrocytes by Flow Cytometry. Mutat. Res. Toxicol. Environ. Mutagen. 464, 195-200. doi: 10.1016/S1383-5718(99)00183-7

Fahey, J. R., and Spitalny, G. L. (1984). Virulent and Nonvirulent Forms of Plasmodium Yoelii Are Not Restricted to Growth Within a Single Erythrocyte Type. Infect. Immun. 44, 151-156. doi: 10.1128/IAI.44.1.151-156.1984

Franke-Fayard, B., Fonager, J., Braks, A., Khan, S. M., and Janse, C. J. (2010). Sequestration and Tissue Accumulation of Human Malaria Parasites: Can We

\section{ACKNOWLEDGMENTS}

The authors would like to thank SIgN Flow Cytometry Core for technical assistance with flow cytometry and SIgN Mouse Core for support in animal husbandry. Scientific input from members of LR and BM labs are also greatly appreciated.

\section{SUPPLEMENTARY MATERIAL}

The Supplementary Material for this article can be found online at: https://www.frontiersin.org/articles/10.3389/fcimb.2021. 680136/full\#supplementary-material

Learn Anything From Rodent Models of Malaria? PloS Pathog. 6, e1001032. doi: 10.1371/journal.ppat.1001032

Gautier, E.-F., Leduc, M., Cochet, S., Bailly, K., Lacombe, C., Mohandas, N., et al. (2018). Absolute Proteome Quantification of Highly Purified Populations of Circulating Reticulocytes and Mature Erythrocytes. Blood Adv. 2, 2646-2657. doi: 10.1182/bloodadvances.2018023515

Gautier, E.-F., Leduc, M., Ladli, M., Schulz, V. P., Lefèvre, C., Boussaid, I., et al. (2020). Comprehensive Proteomic Analysis of Murine Terminal Erythroid Differentiation. Blood Adv. 4, 1464-1477. doi: 10.1182/bloodadvances.2020001652

Gautret, P., Deharo, E., Chabaud, A. G., Ginsburg, H., and Landau, I. (1994). Plasmodium Vinckei Vinckei, P. V. Lentum and P. Yoelii Yoelii: Chronobiology of the Asexual Cycle in the Blood. Parasite 1, 235-239. doi: 10.1051/parasite/ 1994013235

Gruszczyk, J., Kanjee, U., Chan, L.-J., Menant, S., Malleret, B., Lim, N. T. Y., et al. (2018). Transferrin Receptor 1 Is a Reticulocyte-Specific Receptor for Plasmodium Vivax. Science 359, 48-55. doi: 10.1126/science.aan1078

Gunalan, K., Gao, X., Yap, S. S. L., Huang, X., and Preiser, P. R. (2013). The Role of the Reticulocyte-Binding-Like Protein Homologues of Plasmodium in Erythrocyte Sensing and Invasion. Cell. Microbiol. 15, 35-44. doi: 10.1111/ cmi. 12038

Hojo-Souza, N. S., de Azevedo, P. O., de Castro, J. T., Teixeira-Carvalho, A., Lieberman, J., Junqueira, C., et al. (2020). Contributions of IFN- $\gamma$ and Granulysin to the Clearance of Plasmodium Yoelii Blood Stage. PloS Pathog. 16, e1008840. doi: 10.1371/journal.ppat.1008840

Imai, T., Ishida, H., Suzue, K., Hirai, M., Taniguchi, T., Okada, H., et al. (2013). $\mathrm{Cd} 8+\mathrm{T}$ Cell Activation by Murine Erythroblasts Infected With Malaria Parasites. Sci. Rep. 3, 1572. doi: 10.1038/srep01572

Iyer, J., Grüner, A. C., Rénia, L., Snounou, G., and Preiser, P. R. (2007). Invasion of Host Cells by Malaria Parasites: A Tale of Two Protein Families. Mol. Microbiol. 65, 231-249. doi: 10.1111/j.1365-2958.2007.05791.x

Janse, C. J., Franke-Fayard, B., Mair, G. R., Ramesar, J., Thiel, C., Engelmann, S., et al. (2006). High Efficiency Transfection of Plasmodium Berghei Facilitates Novel Selection Procedures. Mol. Biochem. Parasitol. 145, 60-70. doi: 10.1016/ j.molbiopara.2005.09.007

Jarra, W., and Brown, K. N. (1989). Invasion of Mature and Immature Erythrocytes of CBA/Ca Mice by a Cloned Line of Plasmodium Chabaudi Chabaudi. Parasitology 99, 157-163. doi: 10.1017/s0031182000058583

Joice, R., Nilsson, S. K., Montgomery, J., Dankwa, S., Egan, E., Morahan, B., et al. (2014). Plasmodium Falciparum Transmission Stages Accumulate in the Human Bone Marrow. Sci. Transl. Med. 6, 244re5. doi: 10.1126/scitranslmed. 3008882

Junqueira, C., Barbosa, C. R. R., Costa, P. A. C., Teixeira-Carvalho, A., Castro, G., Sen Santara, S., et al. (2018). Cytotoxic CD8+ T Cells Recognize and Kill Plasmodium Vivax-Infected Reticulocytes. Nat. Med. 24, 1330-1336. doi: 10.1038/s41591-018-0117-4

Killick-Kendrick, R., and Warben, M. (1968). Primary Exoerythrocytic Schizonts of a Mammalian Plasmodium as a Source of Gametocytes. Nature 220, $191-$ 192. doi: $10.1038 / 220191 \mathrm{a} 0$

Lee, R. S., Waters, A. P., and Brewer, J. M. (2018). A Cryptic Cycle in Haematopoietic Niches Promotes Initiation of Malaria Transmission and Evasion of Chemotherapy. Nat. Commun. 9, 1689. doi: 10.1038/s41467-01804108-9 
Liu, J., Guo, X., Mohandas, N., Chasis, J. A., and An, X. (2010). Membrane Remodeling During Reticulocyte Maturation. Blood 115, 2021-2027. doi: 10.1182/blood-2009-08-241182

Li, J., Zhang, Y., Sullivan, M., Hong, L., Huang, L., Lu, F., et al. (2007). Typing Plasmodium Yoelii Microsatellites Using a Simple and Affordable Fluorescent Labeling Method. Mol. Biochem. Parasitol. 155, 94-102. doi: 10.1016/ j.molbiopara.2007.06.003

Malleret, B., Li, A., Zhang, R., Tan, K. S. W., Suwanarusk, R., Claser, C., et al. (2015). Plasmodium Vivax: Restricted Tropism and Rapid Remodeling of CD71-positive Reticulocytes. Blood 125, 1314-1324. doi: 10.1182/blood-2014-08-596015

Malleret, B., Sahili, A. E., Tay, M. Z., Carissimo, G., Ong, A. S. M., Noverna, W., et al. (2021). Plasmodium vivax Binds Host CD98hc (SLC3A2) to Enter Immature Red Blood Cells. Nat. Microbiol. In press. doi: 10.1038/s41564-021-00939-3

Malleret, B., Xu, F., Mohandas, N., Suwanarusk, R., Chu, C., Leite, J. A., et al. (2013). Significant Biochemical, Biophysical and Metabolic Diversity in Circulating Human Cord Blood Reticulocytes. PloS One 8, e76062. doi: 10.1371/journal.pone.0076062

Martín-Jaular, L., Elizalde-Torrent, A., Thomson-Luque, R., Ferrer, M., Segovia, J. C., Herreros-Aviles, E., et al. (2013). Reticulocyte-Prone Malaria Parasites Predominantly Invade CD71hi Immature Cells: Implications for the Development of an In Vitro Culture for Plasmodium Vivax. Malar. J. 12, 434. doi: 10.1186/1475-2875-12-434

Naidu, R., Chu, T. T., Tripathi, J., Hu, Y., Subramanian, G., Tong, J. X., et al. (2019). Reticulocyte Infection Leads to Altered Behaviour, Drug Sensitivity and Host Cell Remodelling by Plasmodium Falciparum. bioRxiv 862169, 1-31. doi: $10.1101 / 862169$

Obaldia, N., Meibalan, E., Sa, J. M., Ma, S., Clark, M. A., Mejia, P., et al. (2018). Bone Marrow Is a Major Parasite Reservoir in Plasmodium Vivax Infection. mBio 9, e00625-e00618. doi: 10.1128/mBio.00625-18

Otsuki, H., Kaneko, O., Thongkukiatkul, A., Tachibana, M., Iriko, H., Takeo, S., et al. (2009). Single Amino Acid Substitution in Plasmodium Yoelii Erythrocyte Ligand Determines Its Localization and Controls Parasite Virulence. Proc. Natl. Acad. Sci. 106, 7167-7172. doi: 10.1073/pnas.0811313106

Pasvol, G., Weatherall, D. J., and Wilson, R. J. M. (1980). The Increased Susceptibility of Young Red Cells to Invasion by the Malarial Parasite Plasmodium Falciparum. Br. J. Haematol. 45, 285-295. doi: 10.1111/j.1365-2141.1980.tb07148.x

Srivastava, A., Creek, D. J., Evans, K. J., De Souza, D., Schofield, L., Müller, S., et al. (2015). Host Reticulocytes Provide Metabolic Reservoirs That Can Be Exploited by Malaria Parasites. PloS Pathog. 11, e1004882. doi: 10.1371/journal.ppat.1004882

Swardson-Olver, C. J., Dawson, T. C., Burnett, R. C., Peiper, S. C., Maeda, N., and Avery, A. C. (2002). Plasmodium Yoelii Uses the Murine Duffy Antigen
Receptor for Chemokines as a Receptor for Normocyte Invasion and an Alternative Receptor for Reticulocyte Invasion. Blood 99, 2677-2684. doi: 10.1182/blood.V99.8.2677

Tao, Z.-Y., Xia, H., Cao, J., and Gao, Q. (2011). Development and Evaluation of a Prototype non-Woven Fabric Filter for Purification of Malaria-Infected Blood. Malar. J. 10, 251. doi: 10.1186/1475-2875-10-251

Thakre, N., Fernandes, P., Mueller, A.-K., and Graw, F. (2018). Examining the Reticulocyte Preference of Two Plasmodium Berghei Strains During BloodStage Malaria Infection. Front. Microbiol. 9, 166. doi: 10.3389/fmicb.2018.00166

Venugopal, K., Hentzschel, F., Valkiūnas, G., and Marti, M. (2020). Plasmodium Asexual Growth and Sexual Development in the Haematopoietic Niche of the Host. Nat. Rev. Microbiol. 18, 177-189. doi: 10.1038/s41579-019-0306-2

Viens, P., Chevalier, J. L., Sonea, S., and Yoeli, M. (1971). The Effect of Reticulocytosis on Plasmodium Vinckei Infection in White Mice. Action of Phenylhydrazine and of Repeated Bleedings. Can. J. Microbiol. 17, 257-261. doi: $10.1139 / \mathrm{m} 71-043$

Vigário, A. M., Belnoue, E., Cumano, A., Marussig, M., Miltgen, F., Landau, I., et al. (2001). Inhibition of Plasmodium Yoelii Blood-Stage Malaria by Interferon Alpha Through the Inhibition of the Production of its Target Cell, the Reticulocyte. Blood 97, 3966-3971. doi: 10.1182/blood.v97.12.3966

Weiss, W. R., Good, M. F., Hollingdale, M. R., Miller, L. H., and Berzofsky, J. A. (1989). Genetic Control of Immunity to Plasmodium Yoelii Sporozoites. J. Immunol. 143, 4263-4266.

Wilson, M. C., Trakarnsanga, K., Heesom, K. J., Cogan, N., Green, C., Toye, A. M. et al. (2016). Comparison of the Proteome of Adult and Cord Erythroid Cells, and Changes in the Proteome Following Reticulocyte Maturation. Mol. Cell. Proteomics 15, 1938-1946. doi: 10.1074/mcp.M115.057315

Zuzarte-Luis, V., Mota, M. M., and Vigário, A. M. (2014). Malaria Infections: What and How Can Mice Teach Us. J. Immunol. Methods 410, 113-122. doi: 10.1016/j.jim.2014.05.001

Conflict of Interest: The authors declare that the research was conducted in the absence of any commercial or financial relationships that could be construed as a potential conflict of interest.

Copyright (c) 2021 Leong, Lee, Rénia and Malleret. This is an open-access article distributed under the terms of the Creative Commons Attribution License (CC BY). The use, distribution or reproduction in other forums is permitted, provided the original author(s) and the copyright owner(s) are credited and that the original publication in this journal is cited, in accordance with accepted academic practice. No use, distribution or reproduction is permitted which does not comply with these terms. 